\title{
THE BEHAVIOR OF THE SUPPORT OF SOLUTIONS OF THE EQUATION OF NONLINEAR HEAT CONDUCTION WITH ABSORPTION IN ONE DIMENSION \\ BY \\ BARRY F. KNERR
}

\begin{abstract}
ABSTRACr. We consider the Cauchy problem in one space dimension for a nonlinear degenerate parabolic partial differential equation. The connectedness of the support of the solution and estimates of the growth of the support as $t \rightarrow \infty$ are established.
\end{abstract}

1. Introduction. In this paper we consider the parabolic Cauchy problem

$$
\begin{gathered}
u_{t}=[\varphi(u)]_{x x}-\psi(u) \text { in } R^{1} \times(0, \infty), \\
u(x, 0)=u_{0}(x) \text { in } R^{1},
\end{gathered}
$$

where $u_{0}(x)$ is a bounded continuous nonnegative function whose support is a bounded interval. The functions $\varphi$ and $\psi$ are smooth nonnegative functions satisfying $\varphi(0)=\varphi^{\prime}(0)=\psi(0)=0$, and $\varphi^{\prime}(u), \varphi^{\prime \prime}(u), \psi^{\prime}(u)>0$ if $u>0$. Thus equation (1.1) is nonlinear and degenerates when $u=0$. According to [8], equation (1.1) occurs in the theory of nonlinear heat conduction with absorption.

Existence and uniqueness of continuous bounded nonnegative solutions possessing bounded weak derivatives $\partial \varphi(u) / \partial x$ was established in [8].

If $\psi \equiv 0$ then (1.1) is called the porous medium equation and this equation has been studied by many authors [1]-[5], [7]-[15], [17] $[21]$. The special case

$$
u_{t}=\left(u^{\mu}\right)_{x x}-C u^{\nu}
$$

where $C>0, \mu>1$, and $\nu>0$ has been studied in [9].

$\S 2$ is devoted to stating the assumptions about the functions $\varphi$ and $\psi$ in (1.1) and defining a useful transformation of the solution $u$. Some of our main results for problem (1.1), (1.2) are given in \$3. There we establish the connectedness of the open set $P[u]=\left\{(x, t) \in R^{2} \times(0, \infty) \mid u(x, t)>0\right\}$. We also prove that, under certain conditions on $\varphi$ and $\psi$, the open set $P[u] \cap\{t=\tau\}$ is an interval $\left(\zeta_{1}(\tau), \zeta_{2}(\tau)\right)$ for each $\tau>0$, where the functions $\zeta_{i}$ belong to $C^{0}[0, \infty) \cap C^{0,1}(0, \infty), \zeta_{1} \downarrow$, and $\zeta_{2} \uparrow$. In the special case

Received by the editors March 8, 1977.

AMS (MOS) subject classifications (1970). Primary 35K15, 35K55; Secondary $35 \mathrm{~B} 99$.

Key words and phrases. Nonlinear, parabolic, degenerate, nonlinear heat conduction with absorption, porous medium. 
(1.3) the conditions on $\varphi$ and $\psi$ are that $\mu>1$ and $\nu>1$. It was proved in [8] that $P[u]$ is a bounded subset of $R^{1} \times(0, \infty)$ for $\mu>1$ and $0<\nu<1$.

It follows from results established in [9] that in the special case (1.3) the functions $\zeta_{i}(\tau)$ are bounded if $1 \leqslant v<\mu$ and unbounded if $v>\mu$. In $\S 4$ of this paper we treat the borderline case $\nu=\mu$ for equation (1.3). We prove that the functions $\left|\zeta_{i}(\tau)\right|(i=1,2)$ approach infinity faster than $\sqrt{\ln t}$ but slower than $\sqrt{t^{1 / 2}}$ as $t \rightarrow \infty$.

In [15] it was shown that for the porous medium equation the interface functions $\left|\zeta_{i}(\tau)\right|$ become infinite as $t \rightarrow \infty$ and growth estimates were derived. For the special case

$$
u_{t}=\left(u^{\mu}\right)_{x x}
$$

it was shown that $\left|\zeta_{i}(\tau)\right| \rightarrow \infty$ like $t^{1 /(1+\mu)}$ as $t \rightarrow \infty$. In view of the results of [7], [8], and [9], the addition of the term $-C u^{\nu}$ has a profound effect on the growth of the interface curves for $\nu \in(0, \mu)$. Indeed, for equation (1.3) with $0<\nu<\mu$ the interface curves are bounded. In $\$ 5$ we derive growth estimates as $t \rightarrow \infty$ for the interface in the problem (1.1), (1.2) under conditions on $\varphi$ and $\psi$ which correspond to $\nu>\mu$ in the special case (1.3).

2. Preliminaries. Consider the parabolic equation

$$
u_{t}=[\varphi(u)]_{x x}-\psi(u) \text { in } G \equiv R^{1} \times(0, \infty) .
$$

We assume throughout this paper that the following conditions are met by the functions $\varphi$ and $\psi$, and the inverse function of $\varphi$, denoted by $\Phi$ :

$$
\begin{gathered}
\Phi(v) \in C^{\alpha}[0, M] \quad \text { for all } M>0 \quad(0<\alpha<1), \\
\varphi(u) \in C^{1+\alpha_{1}}[0, M] \cap C^{2+\alpha_{1}}[m, M], \text { for all } M>0, \\
\text { for all } m \in(0, M) \quad\left(0<\alpha_{1}<1\right), \\
\varphi^{(k)}(u) \geqslant 0 \text { for } k=0,1,2 \text { when } u>0, \\
\varphi(0)=0 \text { and } \varphi^{\prime}(u)>0 \text { for } u>0, \\
\int_{0}^{1}\left(\frac{\varphi^{\prime}(s)}{s}\right) d s<\infty \text { and } \sup _{s \in(0,1)} \frac{s \varphi^{\prime \prime}(s)}{\varphi^{\prime}(s)}<\infty \\
\psi(u) \in C[0, M] \cap C^{1+\alpha_{2}}[m, M], \text { for all } M>0, \\
\psi^{\prime}(u)>0 \text { for all } m \in(0, M) \quad\left(0<\alpha_{2}<1\right)
\end{gathered}
$$

In this paper we will consider the Cauchy problem for equation (2.1) with the initial condition

$$
u(x, 0)=u_{0}(x) \text { for } x \in R^{1}
$$


About $u_{0}$ we assume that:

$$
\begin{gathered}
u_{0} \in C_{0}\left(R^{1}\right), \\
u_{0}(x)>0 \text { if }|x|<a \text { and } u_{0}(x) \equiv 0 \text { if }|x|>a>0, \\
\varphi\left(u_{0}\right) \in C^{0,1}\left(R^{1}\right) .
\end{gathered}
$$

Because of the translation invariance of equation (2.1), assumption (2.11) is, of course, equivalent to the assumption that the support of $u_{0}$ consists precisely of a bounded interval. We remark that assumptions $(2.2)-(2.8)$ are valid if we take $\varphi(u)=u^{\mu}$ and $\psi(u)=c u^{\nu}$ where $c>0, \nu>0$ and $\mu>1$. With this choice of functions $\varphi$ and $\psi$, equation (2.1) becomes

$$
u_{t}=\left(u^{\mu}\right)_{x x}-c u^{\nu} \text { in } G \equiv R^{1} \times(0, \infty) \text {. }
$$

It will sometimes be advantageous to change variables in (2.1) through the transformation

$$
v(x, t)=\theta(u(x, t)) \text { in } G
$$

where

$$
\theta(s)=\int_{0}^{s}\left(\frac{\varphi^{\prime}(\xi)}{\xi}\right) d \xi .
$$

This transformation takes equation (2.1) into the equation

$$
v_{t}=\sigma(v) v_{x x}+v_{x}^{2}-k(v) \text { in } G,
$$

where

$$
\begin{gathered}
\sigma(v)=\varphi^{\prime}(u), \\
k(v)=\psi(u)\left(\varphi^{\prime}(u) / u\right), \\
u=\theta^{-1}(v) .
\end{gathered}
$$

With the special functions $\varphi(u)=u^{\mu}$ and $\psi(u)=c u^{\nu}$, equation (2.14) becomes

$$
v(x, t)=(\mu /(\mu-1)) u^{\mu-1}(x, t)
$$

and equation (2.13) becomes

$$
v_{t}=(\mu-1) v v_{x x}+v_{x}^{2}-c^{*} v^{\beta}
$$

where $\beta=(\nu+\mu-2) /(\mu-1)$ and $c^{*}=c \mu((\mu-1) / \mu)^{\beta}$.

Kalashnikov [8] defines a weak solution $u(x, t)$ of the Cauchy problem (2.1), (2.9) as follows: a bounded function $u(x, t)>0$, satisfying a Hölder condition, is said to be a weak solution of (2.1), (2.9) if $u$ satisfies equation (2.9) and if the integral identity

$$
\int_{t_{0}}^{t_{1}} \int_{x_{0}}^{x_{1}}\left[\varphi(u) f_{x x}+u f_{t}-\psi(u) f\right] d x d t-\left.\int_{x_{0}}^{x_{1}} u f d x\right|_{t_{0}} ^{t_{1}}-\left.\int_{t_{0}}^{t_{1}} \varphi(u) f_{x} d t\right|_{x_{0}} ^{x_{1}}=0
$$


holds whenever $0 \leqslant t_{0}<t_{1}$ and $x_{0}<x_{1}$ and for all $f \in C^{1}(\bar{G})$ such that $f_{x x} \in C(\bar{G})$ and $f=0$ for $x=x_{0}, x_{1}$. If $\psi \equiv 0$ then equation (2.1) is called the porous medium equation, and our notion of a weak solution agrees with the definition given in [19]. Kalashnikov [8] proved that the unique weak solution $u(x, t)$ of $(2.1),(2.9)$ is, in fact, given by $\Phi(w)$ (recall that $\Phi=\varphi^{-1}$ ) where $w$ is the pointwise limit in $\bar{G}$ of a monotone decreasing sequence $\left\{w_{n}(x, t)\right\}$ of positive functions in $C\left(\bar{G}_{n}\right) \cap C^{2,1}\left(G_{n}\right)$ which satisfy the equation

$$
\left(w_{n}\right)_{x x}-\Phi\left(w_{n}\right)\left(w_{n}\right)_{t}-\psi\left(\Phi\left(w_{n}\right)\right)=0 \text { in } G_{n} \equiv(-n, n) \times(0, n)
$$

and are bounded uniformly with respect to $n$ as follows:

$$
\sup _{G_{n}} w_{n}(x, t) \leqslant C, \quad \sup _{G_{n}}\left|\frac{\partial}{\partial x} w_{n}(x, t)\right| \leqslant C .
$$

Also, the weak solution $u(x, t)$ is smooth in the open set $\{u>0\}$ (see [8]).

3. The interface. The following lemma is useful for comparing solutions of (2.1) with solutions of the porous medium equation (equation (2.1) with $\psi \equiv 0$ ). The proof is similar to the proofs of comparison theorems in [8], [18], [22]. We include a proof here for completeness.

LEMMA 3.1. Suppose that a function $u(x, t)$ is a weak solution of the Cauchy problem

$$
\begin{gathered}
u_{t}=(\varphi(u))_{x x}-\psi(u) \text { in } G, \\
u(x, 0)=u_{0}(x) \text { in } R^{1}
\end{gathered}
$$

and suppose that another function $\bar{u}(x, t)$ is a solution of the Cauchy problem

$$
\begin{gathered}
\bar{u}_{t}=(\varphi(\bar{u}))_{x x} \text { in } G, \\
\bar{u}(x, 0)=\bar{u}_{0}(x) \quad \text { in } R^{1},
\end{gathered}
$$

where

$$
\bar{u}_{0}(x) \geqslant u_{0}(x) \text { in } R^{1}
$$

Then $\bar{u} \geqslant u$ in $\bar{G}$.

Proof. Recalling the discussion surrounding equation (2.23), we see that there exist sequences $\left\{\bar{w}^{n}\right\}$ and $\left\{w^{n}\right\}$ of positive functions in $C\left(\bar{G}_{n}\right) \cap$ $C^{2,1}\left(G_{n}\right)$ which converge respectively to $\varphi(\bar{u})$ and $\varphi(u)$ in $G$, and such that $\bar{w}^{n}>w^{n}$ on the parabolic boundary of $G_{n}$. In fact, the boundary data can be chosen so that $\bar{w}^{n} \in C^{2,1}\left(\bar{G}_{n}\right)$ (see [16]). Letting $\mathcal{L}$ denote the nonlinear operator given by $\ell_{z}=z_{x x}-\Phi^{\prime}(z) z_{t}$ we see, from equation (2.23) that $\mathcal{L}\left(w^{n}\right) \geqslant 0$ in $G_{n}$ and $\mathcal{L}\left(\bar{w}^{n}\right)=0$ in $G_{n}$. 
One then easily deduces that the inequality

$$
P_{x x}^{n}-a^{n}(x, t) P^{n}-\Phi^{\prime}\left(w^{n}(x, t)\right) P_{t}^{n}>0 \text { in } G^{n}
$$

holds for $P^{n}=w^{n}-\bar{w}^{n}$ and

$$
a^{n}(x, t)=\bar{w}_{t}^{n}(x, t) \int_{0}^{1} \Phi^{\prime \prime}\left(\theta \bar{w}^{n}(x, t)+(1-\theta) w^{n}(x, t)\right) d \theta .
$$

The function $a^{n}(x, t)$ is bounded in $G_{n}$, due to (2.3) and the boundedness of $\bar{w}_{t}^{n}$ in $G_{n}$, and, by virtue of (2.4) and (2.5), the coefficient of $P_{t}^{n}$ in (3.6) is negative and bounded away from zero. Set $Q^{n}=e^{-\alpha t} P^{n}$ where $\alpha$ is a positive constant which we will choose sufficiently large. From inequality (3.6) we get the inequality

$$
\alpha_{n}(x, t) Q_{x x}^{n}+\beta_{n}(x, t) Q^{n}-Q_{t}^{n}>0 \text { in } G_{n}
$$

where $\alpha_{n}(x, t)=\left(\Phi^{\prime}\left(w^{n}(x, t)\right)\right)^{-1}$ and where $\beta_{n}(x, t)=-a^{n}(x, t) / \alpha_{n}(x, t)$ - $\alpha$. Thus, for sufficiently large $\alpha>0$ we have the inequality

$$
\beta_{n}(x, t)<0 \text { in } G_{n} \text {. }
$$

Since $Q^{n}<0$ on the parabolic boundary of $G_{n}$, it follows from the maximum principle (see, for example, [6]) that $Q^{n}<0$ in $G^{n}$ and therefore that

$$
w^{n}(x, t)<\bar{w}^{n}(x, t) \text { in } G_{n} \text {. }
$$

Letting $n \rightarrow \infty$ produces the desired conclusion.

Now recall that $(-a, a)$ is the support of $u_{0}$ and let $N=\sup \theta\left(u_{0}\right)$. Then, Theorem 8.4 and Corollary 9.3 [15] and Lemma 3.1 together give the following result.

THEOREM 3.2. If $u(x, t)$ is a weak solution of (2.1), (2.9), then $u(x, t)=0$ if $t \geqslant 0$ and $|x| \geqslant a+2 \sqrt{N t}$. If $u$ is a weak solution of (2.13), (2.9) then $u(x, t)=0$ if $t \geqslant 1$ and $|x| \geqslant \theta t^{1 /(1+\mu)}$ where $\theta>0$ is a constant depending on $u_{0}$ and $\mu$.

If, for arbitrary constants $\tau>0$ we apply Theorem 3.2 to the function $\tilde{u}(x, t)=u(x, t+\tau)$, it is easy to see that if the support of $u(\cdot, t)$ is monotone increasing in $t$, then the boundary of the support consists of Hölder continuous monotone functions of $t$ (exponent $\frac{1}{2}$ ). Since a better result is forthcoming (see Theorem 3.9) we shall not prove this.

Another immediate consequence of Lemma 3.1 is the following. Recall that $v \equiv \theta(u)$ (see (2.14)). In this theorem we do not necessarily assume that the support of $u$ is the interval $(-a, a)$.

THEOREM 3.3. If for some $x_{0} \in R^{1}$,

$$
v(x, 0)<b\left(x-x_{0}\right)^{2} \text { for }\left|x-x_{0}\right|<\delta
$$

for some constants $b, \delta>0$, then there exists a constant $\tau>0$ such that $u\left(x_{0}, t\right)=0$ for $0<t<\tau$. 
Proof. Theorem 8.2 of [15] establishes this result in the case $x_{0}= \pm a$. However, a careful reading of that proof shows that the result is valid for any $x_{0} \in R^{1}$. Now use Lemma 3.1.

Let $\Psi(s)=\psi(\Phi(s))$ and let $f(s)=\Psi(s) /\left(s \Phi^{\prime}(s)\right)$.

Lemma 3.4. Suppose $f(s) \in L^{\infty}(0,1)$. If $\left(x_{0}, \tau\right) \in \overline{P[u]}$ and if $u\left(x_{0}, \tau\right)>0$, then $u\left(x_{0}, t\right)>0$ for all $t \geqslant \tau$.

Proof. Since $f$ is bounded, it follows that $\int_{0}^{1}\left(\Phi^{\prime}(s) / \Psi(s)\right) d s=\infty$. It is possible, therefore, to define a function $q(t)$ by the equation

$$
\int_{q(t)}^{1} \frac{\Phi^{\prime}(s)}{\Psi(s)} d s=t \text { for } t>0 .
$$

We also define a function $z(x, t)$ by

$$
z(x, t)=\gamma q(t) e^{-\lambda t} \sin \left(\beta\left(x-x_{0}\right)+\pi / 2\right)
$$

where $\gamma, \lambda$ and $\beta$ are positive constants which will be specified later.

Let $R$ denote the rectangle

$$
\left(x_{0}-\frac{\pi}{2 \beta}, x_{0}+\frac{\pi}{2 \beta}\right) \times\left(\tau, t_{1}\right)
$$

and let $\Gamma$ denote its lower and lateral boundary. Also let $\mathcal{L}$ denote the nonlinear operator given by

$$
\mathcal{L}_{\eta}=\eta_{x x}-\Phi^{\prime}(\eta) \eta_{t}-\Psi(\eta)
$$

which occurred previously in equation (2.23). It can easily be checked that

$$
\mathcal{L}_{z}=z\left[\left(\lambda \Phi^{\prime}(z)-\beta^{2}\right)+\Phi^{\prime}(z)(f(q)-f(z))\right]
$$

Because of (2.4), we know that $\Phi^{\prime \prime}<0$ and that $\Phi^{\prime}(z) \geqslant \Phi^{\prime}(\sup z)>\Phi^{\prime}(\gamma)>$ 0 . We shall now choose our parameters $\beta, \lambda$ and $\gamma$ so that $\mathcal{L}_{z}>0$ in $R$. First choose $\beta$ so large that

$$
w(x, \tau)>0 \text { for } x_{0}-\frac{\pi}{2 \beta} \leqslant x \leqslant x_{0}+\frac{\pi}{2 \beta} .
$$

This is possible because, by hypothesis, $w$ is continuous, and pointwise at $\left(x_{0}, \tau\right)$. Next, choose $\gamma \leqslant 1$ so small that

$$
z(x, \tau) \leqslant \gamma<w(x, \tau) \text { for } x \in\left[x_{0}-\frac{\pi}{2 \beta}, x_{0}+\frac{\pi}{2 \beta}\right] .
$$

Now, since $f$ is bounded on $(0,1)$ and $\Phi^{\prime}(z)$ is bounded below by a positive constant independent of $\lambda$, we can clearly choose $\lambda$ so large that

$$
\mathcal{L}_{z}>0 \text { in } \bar{R} / \Gamma \text {. }
$$

Indeed, by equation (3.14) we have

$$
\mathcal{L} z=z\left[\left(\frac{1}{2} \lambda \Phi^{\prime}(z)-\beta^{2}\right)+\Phi^{\prime}(z)\left(\frac{1}{2} \lambda+f(q)-f(z)\right)\right]
$$


and we can first choose $\lambda$ sufficiently large that the first term on the right is nonnegative in $R$, and then choose $\lambda$ still larger, if necessary, so that the second term is nonnegative in $R$.

Now, using the fact that $z(x, t)=0$ if $x=x_{0} \pm \pi / 2 \beta$ we have the relation

$$
z \leqslant w \text { on } \Gamma \text {. }
$$

Theorem 5 [8] then implies that

$$
z \leqslant w \text { in } R
$$

and therefore that

$$
u\left(x_{0}, t\right)>0 \text { for } \tau \leqslant t \leqslant t_{1} .
$$

The result then follows from the arbitrariness of $t_{1}$.

The comparison function $z(x, t)$ defined by equation (3.12), and the main idea in the above proof are both due to Kalashnikov [8].

REMARK 3.5. If $\varphi(s)=s^{\mu}$ and $\psi(s)=c s^{\nu}$ then $f(s)=c \mu s^{(\nu-1) / \mu}$, and Lemma 3.4 is applicable if $\nu \geqslant 1$ and $\mu>0$. Since we are only interested in $\mu>1$ (see the paragraph containing equation (2.13)) the essential restriction here is that $\nu \geqslant 1$. Theorem 10 of [8] shows that Lemma 3.4 fails if $\nu<1$.

THEOREM 3.6. If for all $s \in\left(0, \sup v\right.$ ) we have $k^{\prime}(s)>0$ (where $k$ is defined by equation (2.18)), then, for the function $v(x, t)$ defined by equation (2.14), the following estimate holds:

$$
\left|\frac{\partial}{\partial x} v\right| \leqslant C(\tau) \text { on } P[u] \cap\{t>\tau\} .
$$

If $\sup |(\partial / \partial x) v(x, 0)|<\infty$, then

$$
\left|\frac{\partial}{\partial x} v\right| \leqslant C \text { on } P[u]
$$

where $C$ depends on $\sup |(\partial / \partial x) v(x, 0)|$.

Proof. This is a straightforward generalization of Aronson's proof of this very useful result in the case $\psi \equiv 0$ (see [3]). In fact, if we merely follow Aronson's argument without change, we find that the only difference is that an extra term, $\left(-k^{\prime}\right)$ times a nonnegative factor, is acquired in Aronson's inequalities, and this term can be deleted on the basis of its sign alone.

REMARK 3.7. If $\varphi(u)=u^{\mu}$ and $\psi(u)=c u^{\nu}$, then $k(s)=c^{*} s^{\beta}$ where $\beta=(\nu$ $+\mu-2) /(\mu-1)$ (see equation (2.21)). Thus $\left|v_{x}\right|$ is bounded if $\nu \geqslant 2-\mu$ and $\mu>1$. Kalashnikov [9], using the same technique, proved this and a better result in this special case, namely, that $\left|(\partial / \partial x) u^{\sigma}\right|$ is bounded, where $\sigma=(\mu-\nu) / 2$ if $\nu<2-\mu$.

Recall that $P[u] \equiv\{(x, t) \in G \mid u(x, t)>0\}$ and suppose that the functions $\varphi$ and $\psi$ satisfy conditions (2.2)-(2.8). Then we have the following theorem. 
THEOREM 3.8. For any constant $0<\tau<\infty$ the open set $P_{\tau}[u] \equiv P[u] \cap\{0$ $<t<\tau\}$ is connected. Also for $\tau<{ }^{\prime} \infty, P_{\tau}[u]$ is bounded.

Proof. Let $\tau \in(0, \infty)$ and suppose that $P_{\tau}[u]$ contains two components $Q_{1}$ and $Q_{2}$. First we shall prove that $\bar{Q}_{i} \cap\{t=0\}$ is nonempty for $i=1,2$. Suppose, therefore, that $\bar{Q}_{i} \cap\{t=0\}=\varnothing$ for $i=1$ or for $i=2$. Since $\bar{Q}_{i}$ is compact, by Theorem 3.2, it follows that the function $w(x, t) \equiv \varphi(u(x, t))$ must attain a positive maximum at some point $\left(x_{0}, t_{0}\right) \in \bar{Q}_{i}$. However, the function $w(x, t)$ satisfies the parabolic inequality $\left(1 / \Phi^{\prime}(w)\right) w_{x x}-w_{t}>0$ in $Q_{i}$, so, by the maximum principle it follows that $\left(x_{0}, t_{0}\right) \in \partial Q_{i}$. There are no boundary points of $Q_{i}$ on the line $\{t=0\}$ by hypothesis, so therefore $t_{0}>0$. Moreover, since $w\left(x_{0}, t_{0}\right)>0$ it follows that there is a ball $B$ centered at $\left(x_{0}, t_{0}\right)$ and contained in $P[u]$ so that $B^{*} \equiv B \cap\left\{t<t_{0}\right\} \subset Q_{i}$. Applying the maximum principle again, we derive another contradiction. This proves that $\bar{Q}_{i} \cap\{t=0\} \neq \varnothing$ for $i=1,2$.

We now prove that $\partial Q_{i} \cap\left(a_{1}, a_{2}\right)$ is nonempty for $i=1,2$. If $\partial Q_{i}(i=1,2)$ intersects the line $\{t=0\}$ only at one or both of the points $\left\{a_{1}, a_{2}\right\}$, then we can derive the same contradiction derived above with the aid of the maximum principle.

Thus $\partial Q_{i} \cap\left(a_{1}, a_{2}\right) \neq \varnothing$ for $i=1,2$. But then, because $u \in C(\bar{G})$ and because each $Q_{i}$ is maximal, it follows that $\left(a_{1}, a_{2}\right) \subset \partial Q_{i}$ for $i=1,2$. But then, again by maximality, $Q_{1}=Q_{2}$. This proves that $P_{\tau}[u]$ is connected for each $\tau \in(0, \infty)$. Thus $P[u]$ is also connected. The final assertion follows from Theorem 3.2.

THEOREM 3.9. If the hypotheses of Lemma 3.4 and Theorem 3.6 are fulfilled, then there exist functions $\zeta_{i}(t) \in C[0, \infty) \cap C^{0,1}(0, \infty)(i=1,2)$ such that $\zeta_{1}(t) \searrow, \zeta_{2}(t) \nearrow, \zeta_{1}(0)=-a, \zeta_{2}(0)=a$ and

$$
P[u]=\left\{(x, t) \in G \mid \zeta_{1}(t)<x<\zeta_{2}(t)\right\} .
$$

If $\left|v_{x}(x, 0)\right|_{L^{\infty}\left(R^{\prime}\right)}<\infty$, then $\zeta_{i} \in C^{0,1}[0, \infty)$ for $i=1,2$.

Proof. Let $I_{\tau}$ denote the open set $P[u] \cap\{t=\tau\}$ where $\tau \in(0, \infty)$ and let $\left(x_{1}, \tau\right),\left(x_{2}, \tau\right) \in I_{\tau}$. We will first show that $I_{\tau}$ is an interval. Because $P_{\tau}[u] \equiv$ $P[u] \cap\{t<\tau\}$ is connected (Theorem 3.8) it follows that there exist continuous arcs $\beta_{1}$ and $\beta_{2}$, connecting $\left(x_{1}, \tau\right)$ and $\left(x_{2}, \tau\right)$ to the interval $\left(a_{1}, a_{2}\right)$ lying on $\{t=0\}$, and these arcs must lie in $P_{\tau}[u]$ except at the initial and terminal points. Indeed, this is easily seen by first connecting points $\left(x_{i}^{*}, \tau^{*}\right)(i=1,2)$ to $\left(a_{1}, a_{2}\right)$ where $\tau^{*}<\tau$ and $\left(x_{i}^{*}, \tau^{*}\right)$ is sufficiently close to $\left(x_{i}, \tau\right)(i=1,2)$. But then there clearly exists a continuous arc $\beta$ lying in $P_{\tau}[u] \cup\left(a_{1}, a_{2}\right)$ connecting $\left(x_{1}, \tau\right)$ to $\left(x_{2}, \tau\right)$. Since $\beta$ is continuous it follows that for any $x \in\left(x_{1}, x_{2}\right)$ there must be a point $\left(x, t_{0}\right)$ on $\beta$ for some $t_{0} \in[0, \tau)$. But since $w\left(x, t_{0}\right)>0$, Lemma 3.4 implies that $w(x, \tau)>0$ and 
therefore that $(x, \tau) \in I_{\tau}$. It follows from the arbitrariness of $x, x_{1}$, and $x_{2}$ that $I_{\tau}$ is an interval which we shall denote by $\left(\zeta_{1}(\tau), \zeta_{2}(\tau)\right)$.

As an obvious consequence of Lemma 3.4 it follows that $\zeta_{1}(t) \searrow$ and $\zeta_{2}(t) \lambda$. In order to establish the Lipschitz continuity of $\zeta_{i}(t)(i=1,2)$ let $t_{1}$ and $t_{2}$ be arbitrary nonnegative distinct real numbers. Without loss of generality we can assume that $0<t_{1}<t_{2}<\infty$. Let $u^{*}(x, t)$ denote the solution of the Cauchy problem for the porous medium equation on the half plane $R^{1} \times\left(t_{1}, \infty\right)$ with initial data given by $u^{*}\left(x, t_{1}\right)=u\left(x, t_{1}\right)$. Theorem 3.1 implies that $u(x, t) \leqslant u^{*}(x, t)$ for $(x, t) \in R^{1} \times\left(t_{1}, \infty\right)$. Theorem 6.1 of [15] and Theorem 3.6 then imply that

$$
\zeta_{2}(t)-\zeta_{2}\left(t_{1}\right)<C\left(t-t_{1}\right) \text { for } t>t_{1}
$$

where $C$ is a positive constant depending on $t_{1}$ unless $\left|v_{x}(x, 0)\right|_{L^{\infty}}<\infty$. Using the monotonicity of $\zeta_{2}$ we then have:

$$
0<\zeta_{2}\left(t_{2}\right)-\zeta_{2}\left(t_{1}\right) \leqslant C\left(t_{2}-t_{1}\right)
$$

or

$$
\left|\zeta_{2}\left(t_{2}\right)-\zeta_{2}\left(t_{1}\right)\right| \leqslant C\left|t_{2}-t_{1}\right|
$$

The Lipschitz continuity of $\zeta_{1}(t)$ follows similarly, and the fact that $\zeta_{1}(0)=$ - $a$ and $\zeta_{2}(0)=a$ is an easy consequence of Theorem 3.2 and Lemma 3.4.

Corollary 3.10. If $\varphi(u)=u^{\mu}$ and $\psi(u)=c u^{\nu}$ where $\mu>1$ and $c>0$, then the conclusions of Theorem 3.8 are valid if $\nu>0$ and the conclusions of Theorem 3.9 hold if $\nu \geqslant 1$.

4. A borderline case. In this section we examine the Cauchy problem (2.13), (2.9) with $\mu=\nu$. The cases $\nu>\mu$ and $\nu<\mu$ were considered in [8] and it was there proved that the support is localized in space if $\nu<\mu$ and that it is not if $\nu>\mu$.

During the typing of this manuscript the author received from Robert Kershner [22] a copy of his doctoral dissertation in which, in addition to several other interesting results, it is proved that $P[u]$ cannot be bounded when $\mu=\nu$. In fact, Kershner proves that the support must grow faster than some power of $\ln (\ln t)$. In Theorem 4.1 below we obtain the improved estimate $\left|\zeta_{i}(t)\right|>\theta_{1} \sqrt{\ln t}$ and provide a (sharp) upper estimate as well.

THEOREM 4.1. If $\nu=\mu>1$, then there exist constants $\theta_{1}, \theta_{2}>0$ such that

$$
\theta_{1} \sqrt{\ln t}<\left|\zeta_{i}(t)\right|<\theta_{2} t^{1 /(\mu+1)} \quad(i=1,2)
$$

for $t \geqslant 1$.

Proof. The right inequality is an easy consequence of Lemma 3.1 and Theorem 9.2 of [15]. Equation (2.21) now becomes

$$
v_{t}=(\mu-1) v v_{x x}+v_{x}^{2}-c^{*} v^{2}
$$


where $c^{*}=c(\mu-1)^{2} / \mu>0$. Our strategy is to show that some transformation of an explicit solution of the porous medium equation satisfies an appropriate parabolic inequality so that the maximum principle can be used to show that this transformed solution, whose support grows no slower than $\sqrt{\ln t}$, is dominated by $\theta(u)$ given by (2.14) and (2.20). Now we shall define this comparison function which we will call $v(x, t)$. Let $v(x, t)$ be given by

$$
v(x, t)=s(t) \eta(x, g(s(t))) \text { on } \bar{G}
$$

where

$$
\begin{gathered}
\eta(x, t)=\eta_{0}\left(L x, L^{2} t\right) \text { on } \bar{G} \\
\eta_{0}(x, t)=\frac{\mu}{\mu-1} \lambda^{1-\mu}(t)\left\{1-x^{2} \lambda^{-2}(t)\right\}^{+} \text {on } \bar{G} \\
\lambda(t)=D(t+1)^{1 /(1+\mu)} \text { for } t \geqslant 0, \\
D=\left(\frac{2 \mu(\mu+1)}{\mu-1}\right)^{1 /(1+\mu)}>0, \quad L=\frac{D}{\xi_{1}}
\end{gathered}
$$

The constants $\xi_{1}, \xi_{2}>0$ are chosen so that

$$
\theta(u(x, 0)) \geqslant \xi_{2} \chi\left[-\xi_{1}, \xi_{1}\right](x) \text { on } R^{1},
$$

where $\chi$ is the characteristic function. The functions $s$ and $g$ will be specified later. We remark that the function $\eta_{0}(x, t)$ is due to Barenblatt [4], [5] (see also [15]), and that $\eta$ satisfies

whenever $\boldsymbol{\eta}>0$.

$$
\eta_{t}=(\mu-1) \eta \eta_{x x}+\eta_{x}^{2}
$$

Now we shall specify the functions $s$ and $g$. First, let $\mathcal{L}$ denote the nonlinear operator given by

$$
\ell_{z}=(\mu-1) z z_{x x}+z_{x}^{2}-c^{*} z^{2}-z_{t}
$$

(see (4.1)). Then, from (4.2) and (4.8) we get

$$
e_{v}=\left(s-s^{\prime} g^{\prime}\right) s \eta_{t}-\left[s^{\prime} \eta+c^{*} s^{2} \eta^{2}\right]
$$

where the arguments of $s, g$, and $\eta$ are $t, s(t)$ and $(x, g(s(t)))$, respectively. In order to have $\mathcal{L} v \geqslant 0$ whenever $v>0$ we shall require that $s$ and $g$ be positive and satisfy the conditions:

$$
\begin{gathered}
(d / d t) g(s(t))=s(t) \text { for } t>0, \\
-s^{-2}(t) s^{\prime}(t) \geqslant c^{*} \eta(x, g(s(t))) \text { for } t>0 .
\end{gathered}
$$

Since it is easily seen that $\eta(x, g(s(t)))<\eta(0, g(s(t)))$ for all $(x, t) \in G$, condition (ii) can be replaced by the condition

$$
-s^{-2}(t) s^{\prime}(t)=c_{2}\left(1+L^{2} g(s(t))\right)^{(1-\mu) /(1+\mu)}
$$


where we have used equations (4.3) and (4.4) and when

$$
c_{2}=c^{*}\left(\frac{\mu}{\mu-1} D^{1-\mu}\right) \text {. }
$$

We now define the function $g(w)$ by

$$
1+L^{2} g(w)=\left[\frac{-2 L^{2}}{c_{2}(1+\mu)}\left(\ln \frac{w}{s_{0}}\right)+1\right]^{(1+\mu) / 2} \text { for } w>0
$$

and we define the function $s(t)$ to be the solution of the initial value problem

$$
-s^{-2} s^{\prime}=c_{2}\left[\frac{-2 L^{2}}{c_{2}(1+\mu)}\left(\ln \frac{s}{s_{0}}\right)+1\right]^{(1-\mu) / 2} \text { for } t \geqslant 0
$$

with initial condition

$$
s(0)=s_{0} \equiv\left(\frac{\mu-1}{\mu}\right) \xi_{2} D^{\mu-1}
$$

(see (4.6), (4.7)). It is not difficult to verify formally that conditions (i) and (ii)' above are satisfied by our choice of the functions $g$ and $s$.

We claim that a positive solution $s(t)$ of (4.11), (4.12) exists for all $t>0$, and that $s$ strictly decreases to zero as $t \rightarrow \infty$. Indeed, local existence and uniqueness, for small $t>0$, is standard, and monotonicity is obvious from equation (4.11). To see that $s$ cannot vanish for finite values of $t$ we observe that equation (4.11) implies that

$$
s(t)=s_{0} \exp \left\{-\int_{0}^{t} c_{2} s(\xi) E(\xi) d \xi\right\}
$$

where

$$
E(\xi)=\left[\frac{-2 L^{2}}{c_{2}(1+\mu)}\left(\ln \frac{s(\xi)}{s_{0}}\right)+1\right]^{(1-\mu) / 2} .
$$

The nonvanishing of $s$ then follows from the boundedness of $E$ and equation (4.13). From this observation, the monotonicity of $s$, and equation (4.11) it is obvious that $s$ decreases to zero as $t$ approaches infinity.

We have thus shown that a unique positive solution of (4.11), (4.12) exists and that

$$
s^{\prime}(t)<0 \text { and } s(t)>0 \text { for } t>0
$$

and

$$
\lim _{t \rightarrow \infty} s(t)=0 .
$$

Since conditions (i) and (ii)' hold, we have also established that

$$
\mathcal{L}_{v} \geq 0 \text { when } v>0 \text {. }
$$


Equations (4.2)-(4.5) imply that $v(x, t)>0$ iff $|x|<\zeta(t)$ where

$$
\zeta(t)=\frac{D}{L}\left(1+L^{2} g(s(t))\right)^{1 /(1+\mu)} \text { for } t>0 .
$$

Thus (4.17) can be restated as

$$
\mathcal{L}_{v}>0 \text { if }|x|<\zeta(t) \text { and } t>0 .
$$

We will now show that $v(x, t)<\theta(u(x, t)) \equiv v^{*}(x, t)$ in $G$. Let $\left\{w_{n}\right\}$ be the decreasing sequence of smooth positive functions satisfying (2.23) $-(2.25)$ and converging to $\varphi(u)$. Let $v^{n}=(\theta \circ \Phi)\left(w^{n}\right)$. Then the functions $v^{n}$ satisfy the equation

$$
e v^{n}=0 \text { in } G_{n} \text {. }
$$

For arbitrary $T>0$, let $D_{T}$ be the domain $D_{T}=\{(x, t)|| x \mid<\zeta(t)$ and $0<t<T\}$. Henceforth we assume that $n$ is large enough so that $D_{T} \subset G_{n}$. It is easy to show that (4.19) and (4.20) imply that the function $P^{n}=v^{n}-v$ satisfies the inequality

$$
(\mu-1) v^{n} P_{x x}^{n}+\left(v_{x}^{n}+v_{x}\right) P_{x}^{n}-f(x, t) P^{n}-P_{t}^{n}<0
$$

in $D_{T}$, where

$$
f(x, t)=c^{*}\left(v^{n}(x, t)+v(x, t)\right)-(\mu-1) v_{x x}(x, t) .
$$

Since it is easily verified that $\partial^{2} \eta_{0} / \partial x^{2}<0$ (see (4.4)) it follows that $v_{x x}<0$ and that $f(x, t)>0$ in $D_{T}$. Because $v^{n}>0$ and $v=0$ when $|x|=\zeta(t)$ it follows that $P^{n}>0$ on the lateral boundary of $D_{T}$. Equation (4.10) implies that $g(s(0))=0$ and thus by equations (4.2)-(4.4) we have that

$$
v(x, 0)=s_{0}\left(\frac{\mu}{\mu-1}\right) D^{1-\mu}\left\{1-\frac{L^{2} x^{2}}{D^{2}}\right\}^{+}
$$

so that, by equations (4.6), (4.7) and (4.12) we have

$$
v(x, 0) \leqslant s_{0} \frac{\mu}{\mu-1} D^{1-\mu}=\xi_{2}, \quad x \in R^{1},
$$

and

$$
v(x, 0)=0 \text { for }|x|>D / L=\xi_{1} .
$$

Hence equation (4.7) yields the inequalities

$$
v(x, 0)<v^{*}(x, 0)<v^{n}(x, 0) \text { for } x \in(-n, n) .
$$

Applying the maximum principle we see from (4.21) and the above observations that $P^{n}>0$ in $\bar{D}_{T}$. Letting $n$ approach infinity we see that $v^{*} \geqslant v$ in $D_{T}$ for arbitrary $T>0$ and therefore that

$$
v^{*}(x, t) \geqslant v(x, t) \text { for }|x|<\zeta(t), \quad t \geqslant 0 .
$$

We shall now obtain a lower bound for $\zeta(t)$ as $t \rightarrow \infty$. Equations (4.10) and 
(4.18) together yield

$$
\zeta(t)=\frac{D}{L}\left[\frac{-2 L^{2}}{c_{2}(1+\mu)} \ln \left(\frac{s(t)}{s_{0}}\right)+1\right]^{1 / 2}
$$

or that

$$
\zeta(t)>\text { Const }\left[-\ln \left(\frac{s}{s_{0}}\right)\right]^{1 / 2} \text { for } t>0
$$

Let

$$
\xi(t)=-\ln \left(\frac{s(t)}{s_{0}}\right) \text { for } t>0 .
$$

Equation (4.11) implies that the function $\xi$ satisfies

$$
\xi^{\prime}=B e^{-\xi(t)}(A \xi+1)^{(1-\mu) / 2} \text { for } t>0,
$$

where $B=s_{0} c_{2}$ and $A=2 L^{2} /\left(c_{2}(1+\mu)\right)$. By (4.14) and (4.27) we have that $\xi>0$ and that $\xi(t) \lambda \infty$ as $t \rightarrow \infty$. Hence there exists a positive constant $T^{*}$ such that $e^{\xi(t)}>[\xi(t)]^{(\mu-1) / 2}$ when $t>T^{*}$. Thus we obtain from equation (4.28) the inequality

$$
\xi^{\prime}>\omega e^{-2 \xi} \text { for } t>T^{*}
$$

where $\omega=c_{2} s_{0}\left(A+1 / \xi\left(T^{*}\right)\right)^{(1-\mu) / 2}$ and this immediately implies that

$$
\xi(t)>\frac{1}{2} \ln \left(t-T^{*}\right)+\frac{1}{2} \ln [2 \omega]
$$

for $t>T^{*}$. The result now follows for $t>T^{*}$ from (4.26) and (4.30). The result then follows for $t>1$ by redefining $\theta_{1}$, if necessary, so that $\theta_{1}<\zeta_{1}(0) / \sqrt{\ln T^{*}}$.

5. The growth of the interface as $t \rightarrow \infty$. In this section the functions $\sigma$ and $k$ are again given by equations (2.17) and (2.18) and $u(x, t)$ is a weak solution of (2.1), (2.9).

THEOREM 5.1. Suppose that for some constants $c^{*}>0, \varepsilon>0, \mu>1$ and $2<\beta<2 \mu /(\mu-1)$ such that

$$
\sigma(s)<(\mu-1) s \text { for } 0<s<\varepsilon
$$

and

$$
k(s)<c^{*} s^{\beta} \text { for } 0<s<\varepsilon .
$$

Then there exist positive constants $\theta_{1}$, depending on $\mu$ and $\beta$, and $\theta_{2}$ depending on $\mu$ such that

$$
\theta_{1} t^{((v-\mu) /(v-1)) / 2}<\left|\zeta_{i}(t)\right|<\theta_{2} t^{1 / 2} \quad(i=1,2)
$$

holds for all $t>1$, where $\nu=(\mu-1) \beta-\mu+2$. 
If $\varphi(u)=u^{\mu}$ then we can replace $t^{1 / 2}$ by $t^{1 /(1+\mu)}$ in (5.3).

Proof. The right inequality of (5.3) follows directly from Theorem 3.2. Since we shall prove the left inequality by the same technique used to prove Theorem 4.1, we will only sketch the proof. By Theorem 4 of [8], we may assume without loss of generality that

$$
\bar{v}(x, t) \equiv \theta(u(x, t))<\varepsilon / 2 \text { in } \bar{G}
$$

where $\varepsilon$ is the constant occurring in (5.1) and (5.2). Now let $c$ be given by the condition

$$
c^{*}=c \mu\left(\frac{\mu-1}{\mu}\right)^{\beta} .
$$

As in the proof of Theorem 4.1 it is possible to show that

$$
v(x, t) \leqslant \bar{v}(x, t) \text { for }(x, t) \in \bar{G},
$$

where

$$
v(x, t)=s(t) \eta(x, g(s(t))) \text { in } \bar{G},
$$

and where

$$
\begin{gathered}
g(w)=\frac{1}{L^{2}}\left\{\left[A_{1} \delta\left(w^{2-\beta}-s_{0}^{2-\beta}\right)+1\right]^{1 / \delta}-1\right\} \text { for } w>0, \\
s^{\prime}(t)=-c_{2} s^{\beta}\left[A_{1} \delta\left(s(t)^{2-\beta}-s_{0}^{2-\beta}\right)+1\right]^{1-1 / \delta}, \\
s(0)=s_{0},
\end{gathered}
$$

where $s_{0}$ and $L$ are suitably chosen positive constants and where $c_{2}$ is given by (4.9), $\eta$ is given by (4.3)-(4.6), $\delta=(2+\mu-\nu) /(1+\mu)$ and $A_{1}=L^{2} /\left(c_{2}(\beta\right.$ - 2)). In carrying out the calculations, it is useful to observe that $\delta<0$ and that $1-1 / \delta=(1-\nu) /(2+\mu-\nu)<0$. It is also useful to note that, instead of (4.21), we now get the following inequality for $P^{n}=v-v^{n}$ in $D_{T}$ :

$$
\begin{gathered}
\sigma\left(v^{n}\right) P_{x x}^{n}+\left(v_{x}+v_{x}^{n}\right) P_{x}^{n}-c^{*} v^{\beta}+k\left(v^{n}\right)+(\mu-1) v_{x x} P^{n}-P_{t}^{n} \\
\geqslant\left(\sigma\left(v^{n}\right)-(\mu-1) v^{n}\right) v_{x x} .
\end{gathered}
$$

Using (5.1), (5.2) and (5.4) and the fact that $v_{x x} \leqslant 0$ we get the inequality

$$
\sigma\left(v^{n}\right) P_{x x}^{n}+q_{1}(x, t) P_{x}^{n}-q_{2}(x, t) P^{n}-P_{t}^{n}>0 \text { in } D_{T},
$$

where

$$
q_{1}(x, t)=v_{x}(x, t)+v_{x}^{n}(x, t)
$$

and

$$
q_{2}(x, t)=-(\mu-1) v_{x x}+c^{*} \beta \int_{0}^{1}\left(\theta v+(1-\theta) v^{n}\right)^{\beta-1} d \theta
$$


Because $q_{2}(x, t)$ is bounded, we can apply the maximum principle to the function $Q^{n}(x, t) \equiv e^{-F t} P^{n}(x, t)$ for sufficiently large $F>0$ and conclude that inequality (5.6) is valid. From (5.9) and (5.10) one obtains the estimate

$$
s(t) \leqslant\left[\left(\sigma^{*}-1\right) c_{2} l t+s_{0}^{1-\sigma^{*}}\right]^{1 /\left(1-\sigma^{*}\right)} \text { for } t \geqslant 0
$$

where $l$ is a positive constant and where $\sigma^{*}=\beta+(2-\beta)(1-1 / \delta) \geqslant \beta>$ 2. This estimate, together with (4.16) and (5.8) $-(5.10)$ implies that for $i=1,2$ we have

$$
\zeta_{i}(t) \geqslant \frac{D}{L} P^{1 /(1-\nu)}\left[\left(\sigma^{*}-1\right) c_{2} l t+s_{0}^{1-\sigma^{*}}\right]^{((\nu-\mu) /(\nu-1)) / 2}
$$

for $t \geq 0$, where $P>0$ is a constant, and this establishes the result.

REMARK 5.2. Theorem 9 of [8] gives conditions on $\varphi$ and $\psi$ so that $\left|\zeta_{i}(t)\right| \rightarrow \infty$ as $t \rightarrow \infty$. Although these conditions are not the same as conditions (5.1) and (5.2) of this paper, and the proof is different, nevertheless Theorem 9 of [8] shows that the interface grows faster than some power of $t$ where that power depends upon $\varphi$ and $\psi$. In the special case $\varphi(u)=u^{\mu}$ and $\psi(u)=c u^{\nu}$, the left inequality of (5.3) can also be obtained by a careful reading of the proof of Theorem 9 of [8].

COROLlary 5.3. Suppose that the hypotheses of Theorem 5.1 hold with a constant $\beta \in[2 \mu /(\mu-1), \infty)$. Then, for every $\varepsilon_{0}>0$ there exist a constant $\theta_{1}$ depending on $\varepsilon_{0}$ and a constant $\theta_{2}$ such that

$$
\theta_{1} t^{(1 /(1+\mu))-\varepsilon_{0}} \leqslant\left|\zeta_{i}(t)\right| \leqslant \theta_{2} t^{1 / 2} \quad(i=1,2)
$$

for $t \geqslant 1$, and if $\varphi(u)=u^{\mu}$, then,

$$
\theta_{1} t^{(1 /(1+\mu))-\varepsilon_{0}} \leqslant\left|\zeta_{i}(t)\right| \leqslant \theta_{2} t^{1 /(1+\mu)} \quad(i=1,2)
$$

holds for $t \geqslant 1$.

Proof. Since we assume that (5.2) holds for some $\beta \geqslant 2 \mu /(\mu-1)$, it follows that it holds for each $\beta \in(2,2 \mu /(\mu-1))$. Since $\beta \nearrow 2 \mu /(\mu-1) \Rightarrow \nu \nearrow \mu+2 \Rightarrow \frac{1}{2}(\nu-\mu) /(\nu-1) \nearrow 1 /(1+\mu)$, the result follows.

\section{REFERENCES}

1. D. G. Aronson, Regularity properties of flows through porous media: The interface, Arch. Rational Mech. Anal: 37 (1970), 1-10.

2. $\longrightarrow$ Regularity properties of flows through porous media: A counterexample, SIAM J. Appl. Math. 19 (1970), 299-307.

3. __ Regularity properties of flows through porous media, SIAM J. Appl Math. 17 (1969), $461-467$.

4. G. I. Barenblatt, On one class of solutions of the one-dimensional problem of nonstationary filtration of a gas in a porous medium, Prikl. Mat. Meh. 17 (1953), 739-742.

5. Some nonstationary motion of $a$ liquid and a gas in a porous medium, Prikl. Mat. Meh. 16 (1952), 67-78. 
6. A. Friedman, Partial differential equations of the parabolic type, Prentice-Hall, Inc., Englewood Cliffs, N. J., 1969.

7. M. E. Gurtin and R. C. MacCamy, On the diffusion of biological populations, Math. Biosci. 33 (1977), 35-49.

8. A. S. Kalashnikov, The propagation of disturbances in problems of nonlinear heat conduction with absorption, Ž. Vyčisl. Mat. i Mat. Fiz. 14 (1974), 891-905.

9. state filtration type, Vestnik Moskov. Univ. Ser. I Mat. Meh. 29 (1974), 62-68.

10. __ On uniqueness conditions for the generalized solution of the Cauchy problem for a class of quasilinear degenerate parabolic equations, Differencial'nye Uravnenija 9 (1973), 2207-2212.

11. Cauchy's problem in a class of increasing functions for certain quasilinear degenerate parabolic equations of the second order, Differencial'nye Uravnenija 9 (1973), 682-691.

12. On equations of the nonstationary filtration type in which the perturbation is propagated at infinite velocity, Vestnik Moskov. Univ. Ser. I Mat. Meh. 27 (1972), 45-49.

13. On the occurrence of singularities in the solutions of the equation of nonstationary filtration, Z. Vyčisl. Mat. i Mat. Fiz. 7 (1967), 440-444.

14. S. Kamenomostskaya, The asymptotic behavior of the solution of the filtration equation, Israel J. Math. 14 (1973), 76-87.

15. B. F. Knerr, The porous medium equation in one dimension, Trans. Amer. Math. Soc. 234 (1977), 381-415.

16. O. A. Ladyženskaja, V. A. Solonnikov and N. N. Ural'ceva, Linear and quasilinear equations of the parabolic type, Transl. Math. Monographs, vol. 23, Amer. Math. Soc., Providence, R. I., 1968.

17. M. Muskat, The flow of homogeneous fluids through porous media, McGraw-Hill, New York, 1937.

18. O. A. Oleinik, On some degenerate quasilinear parabolic equations, Sem. dell'Istituto Naz. di Alta Matematica 1962-1963, Oderisi, Gubbio, 1964, pp. 355-371.

19. O. A. Oleinik, A. S. Kalashnikov and Czou Yui-Lin, The Cauchy problem and boundary problems for equations of the type of nonstationary filtration, Izv. Akad. Nauk SSSR Ser. Mat. 22 (1958), 667-704.

20. R. E. Prattle, Diffusion from an instantaneous point source with a concentration-dependent coefficient, Quart. J. Mech. Appl. Math. 12 (1959), 407-409.

21. E. S. Sabinina, On the Cauchy problem for the equation of nonstationary gas filtration in several space variables, Dokl. Akad. Nauk SSSR 136 (1961), 1034-1037.

22. R. Kershner, On some properties of generalized solutions of quasilinear parabolic degenerate equations, dissertation, University of Moscow, 1976. (Russian)

Department of Mathematics, University of Kentucky, LeXington, Kentucky 40506 